Ferrata Storti Foundation

\title{
Association of early disease progression and very poor survival in the GALLIUM study in follicular lymphoma: benefit of obinutuzumab in reducing the rate of early progression
}

Haematologica 2019

Volume 104(6):1202-1208

\section{Correspondence:}

JOHN SEYMOUR

john.seymour@petermac.org

Received: October 14, 2018.

Accepted: December 17, 2018.

Pre-published: December 20, 2018.

doi:10.3324/haematol.2018.209015

Check the online version for the most updated information on this article, online supplements, and information on authorship \& disclosures: www.haematologica.org/content/104/6/1202

(C)2019 Ferrata Storti Foundation

Material published in Haematologica is covered by copyright. All rights are reserved to the Ferrata Storti Foundation. Use of published material is allowed under the following terms and conditions:

https://creativecommons.org/licenses/by-nc/4.0/legalcode. Copies of published material are allowed for personal or internal use. Sharing published material for non-commercial purposes is subject to the following conditions:

https://creativecommons.org/licenses/by-nc/4.0/leǵalcode, sect. 3. Reproducing and sharing published material for commercial purposes is not allowed without permission in writing from the publisher.

\author{
John F. Seymour, ${ }^{1}$ Robert Marcus, ${ }^{2}$ Andrew Davies, ${ }^{3}$ Eve Gallop-Evans, ${ }^{4}$ \\ Andrew Grigg, ${ }^{5}$ Andrew Haynes, ${ }^{6}$ Michael Herold, ${ }^{7}$ Thomas IIImer, ${ }^{8}$ \\ Herman Nilsson-Ehle, ${ }^{9}$ Martin Sökler, ${ }^{10}$ Ulrich Dünzinger, ${ }^{11}$ Tina Nielsen, ${ }^{12}$ \\ Aino Launonen ${ }^{12}$ and Wolfgang Hiddemann ${ }^{13}$
}

${ }^{1}$ Peter MacCallum Cancer Centre, Royal Melbourne Hospital and University of Melbourne, Victoria, Australia; ${ }^{2}$ Kings College Hospital, London, UK; ${ }^{3}$ Cancer Research UK Centre, University of Southampton, UK; ${ }^{4}$ Velindre Cancer Centre, Cardiff, UK; ${ }^{5}$ Austin Hospital, Melbourne, Victoria, Australia; ${ }^{6}$ Nottingham University Hospitals NHS Trust, UK;

${ }^{7} \mathrm{HELIOS}-K$ linikum Erfurt, Germany; ${ }^{8}$ BAG Freiberg-Richter, Jacobasch, IIImer and Wolf, Dresden, Germany; ' ${ }^{S}$ Section of Hematology and Coagulation, Department of Medicine, Sahlgrenska University Hospital, Gothenburg, Sweden; ${ }^{10}$ Eberhard-Karls-University Tübingen, Germany; ${ }^{11}$ Roche Pharma AG, Grenzach-Wyhlen, Germany; ${ }^{12} \mathrm{~F}$. Hoffmann-La Roche Ltd., Basel, Switzerland and ${ }^{13}$ Department of Medicine III, Ludwig-MaximiliansUniversity, Munich, Germany

\section{ABSTRACT}

W e evaluated early disease progression and its impact on overall survival (OS) in previously untreated follicular lymphoma patients in GALLIUM (clinicaltrials.gov identifier: 01332968), and investigated the effect on early disease progression of the two randomization arms: obinutuzumab-based versus rituximab-based immunochemotherapy. Cause-specific Cox regression was used to estimate the effect of treatment on the risk of disease progression or death due to disease progression within 24 months of randomization and to analyze OS in patients with or without disease progression after 24 months. Mortality in both groups was analyzed 6, 12, and 18 months post randomization (median follow up, 41 months). Fewer early disease progression events occurred in obinutuzumab (57 out of 601) versus rituximab (98 out of 601) immunochemotherapy patients, with an average risk reduction of 46.0\% (95\%CI: 25.0-61.1\%; cumulative incidence rate 10.1\% vs. $17.4 \%$ ). At a median post-progression follow up of 22.6 months, risk of mortality increased markedly following a progression event [HR of time-varying progression status, 25.5 (95\% CI: 16.2-40.3)]. Mortality risk was higher the earlier patients progressed within the first 24 months. Age-adjusted HR for OS after 24 months in surviving patients with disease progression versus those without was 12.2 (95\% CI: 5.6-26.5). Post-progression survival was similar by treatment arm. In conclusion, obinutuzumab plus chemotherapy was associated with a marked reduction in the rate of early disease progression events relative to rituximab plus chemotherapy. Early disease progression in patients with follicular lymphoma was associated with poor prognosis, with mortality risk higher after earlier progression. Survival post progression did not seem to be influenced by treatment arm.

\section{Introduction}

Despite the favorable outcomes for patients with previously untreated follicular lymphoma (FL) that are now achievable with the current standard treatment of rituximab (R) plus chemotherapy (R-chemo) followed by R maintenance, $20-35 \%$ of patients still have progressive disease (PD), relapse, or death within two years. ${ }^{1-3}$ 
Patients with early PD have a much poorer survival than those with later disease progression. ${ }^{4,5}$ The glycoengineered anti-CD20 antibody obinutuzumab (GA101; G) has reduced complement-dependent cytotoxicity compared with R, but its direct cell-killing ability, as well as its antibody-dependent cell-mediated cytotoxicity and antibody-dependent cellular phagocytosis, are stronger than those of R., In the recently reported GALLIUM study (clinicaltrials.gov identifier: 01332968) in previously untreated patients with advanced FL, investigatorassessed progression-free survival (PFS) was significantly improved with G-based immunochemotherapy (Gchemo) relative to R-chemo [hazard ratio (HR), 0.68; 95\% confidence interval (CI): 0.54-0.87; $P=0.002]{ }^{8}$

The aims of this exploratory analysis of patients with FL in GALLIUM were to evaluate early PD and its impact on overall survival (OS), and to investigate the impact of $\mathrm{G}$-chemo versus $\mathrm{R}$-chemo treatment on the incidence of early PD.

\section{Methods}

GALLIUM was an open-label, randomized, parallel-group study in which patients with previously untreated grade 1-3a FL were randomized to receive induction therapy with $\mathrm{G}$ or $\mathrm{R}$ plus chemotherapy [cyclophosphamide, doxorubicin, vincristine and prednisone (CHOP), cyclophosphamide, vincristine and prednisone (CVP) or bendamustine] for 6 or 8 cycles, depending on the selected chemotherapy, followed in responders by two years of maintenance with the same antibody. GALLIUM was conducted in accordance with the International Conference on Harmonisation Guidelines for Good Clinical Practice. The protocol was approved by the ethics committees of all participating centers. All patients provided written informed consent. Patient selection, study methods and treatment are described in detail in the Online Supplementary Methods, and elsewhere. ${ }^{9}$

The principal end point in the current exploratory analysis was $\mathrm{PD}$ or death due to PD within 24 months of randomization ("POD24"); "noPOD24" was defined as patients who had completed 24 months of follow up with no PD or death due to PD during that time. Details of analyses to assess PD or suspected transformation are described in the Online Supplementary Appendix. Deaths from causes other than PD during the 24-month window in patients without prior $\mathrm{PD}$, e.g. fatal adverse events or deaths from unrelated causes, contributed as events in noPOD24 mortality, but patients with these events are not included in the description of baseline characteristics for noPOD24 patients.

Analyses were based on investigator assessments and were undertaken using $\mathrm{SAS}^{\otimes} 9.4$ software.

For all randomized patients, the effect of POD24 status on OS was quantified using a time-varying Cox regression model with POD24 status as a time-dependent co-variate. Other co-variates were allocated study treatment and age at randomization; chemotherapy regimen and Follicular Lymphoma International Prognostic Index (FLIPI) score were stratification factors. In this model (and for the calculation of crude death rates), all patients were considered POD24-free until transition to the POD24 group after a POD24 event, or until the end of survival follow up. The effect of the study treatment on the risk of POD24 was estimated by cause-specific Cox regression accounting for POD24 events and censoring for non-PD related deaths within the first 24 months. Analysis was stratified by chemotherapy regimen and FLIPI score.

Overall survival from 4 specified time points ("landmarks"; at
$6,12,18$, and 24 months post randomization) was estimated using Kaplan-Meier (KM) methods. These estimates allow a descriptive, non-randomized comparison of $O S$ in patients with $\mathrm{PD}$ who were still alive versus those with no PD at the landmark. The 24-month landmark analysis was carried out using a Cox regression model, with the same co-variates and stratification factors as the time-dependent model, but with POD24 status as a time-fixed covariate. The risk of a PFS event within 24 months of randomization was also estimated, and the 24-month KM rates were compared across treatment arms.

Post-progression mortality rates in POD24 patients were estimated for all patients and for each treatment arm separately, and for patients who progressed between $0-6,>6-12,>12-18$, and $>18-24$ months from randomization. NoPOD24 mortality rates (overall and by treatment arm) were also calculated.

\section{Results}

\section{Patients' characteristics}

Of 1202 patients with FL randomized in GALLIUM (Online Supplementary Figure S1), 1071 were followed for response until at least 24 months post randomization and evaluated for POD24 status. For the remaining 131 patients, follow up was incomplete (mostly due to study discontinuation): 23 of these patients died from non-PD related causes without prior PD. POD24 events occurred in a total of 155 patients $(12.9 \%)$, of whom 41 died within 24 months of randomization; 916 patients had no POD24 event after at least 24 months of follow up (Online Supplementary Table S1). The data cutoff for this analysis was September 10, 2016, giving a median follow up of 41 months.

Baseline characteristics for POD24 and noPOD24 patients showed some notable differences (Table 1). Compared with noPOD24 patients, POD24 patients were more likely to have the following features: male sex, stage IV disease, grade $3 a$ histology, high FLIPI risk, elevated serum lactate dehydrogenase (LDH), and bulky disease. Relatively more POD24 patients than noPOD24 patients were treated with CVP and fewer were treated with bendamustine. Baseline characteristics for those patients who progressed within six months of randomization are shown in Online Supplementary Table S2.

\section{Progressive disease or death due to progressive disease and progression-free survival by treatment arm}

Progressive disease or death due to progressive disease at 24-months post randomization events occurred in 57 of 601 patients $(9.5 \%)$ treated with G-chemo and 98 of 601 patients $(16.3 \%)$ treated with $\mathrm{R}$-chemo; cumulative incidence rates were $10.1 \%$ (95\% CI: $8-12 \%$ ) and $17.4 \%$ (95\%CI: 14-20\%), respectively. The average HR-based reduction in the risk of a POD24 event with G-chemo relative to R-chemo was $46.0 \%$ (95\% CI: 25.0-61.1\%; $P=0.0003$, Gray's test for equality) (Figure 1). The risk of a PFS event in the 24 months after randomization was 18.9\% (95\% CI: $15.9-22.4 \%$ ) for R-chemo and 12.5\% (95\%CI: $10.1-15.6 \%$ ) for G-chemo; the relative risk reduction for PFS events was $33.9 \%$ (95\% CI: 12.8$49.8 \%$ ) (Figure 1). In the noPOD2 4 group, with a median subsequent follow up of 24 months, 88 out of 916 patients had $\mathrm{POD}$ events after the 24 -month time point [G-chemo, 40 out of 478 (8.4\%); R-chemo, 48 out of 438 $(11.0 \%)]$. 
Post-progression survival and mortality by treatment arm

With a median follow up of 41 months, 56 out of 155 POD24 patients have died: 40 due to PD (G-chemo, $n=15$; R-chemo, $n=25)$ and 16 for other reasons ( $n=4$ and 12, respectively) (Online Supplementary Table S3). In addition, 16 out of 916 noPOD24 patients have died: one related to PD (allocated to the G-chemo arm but no study treatment received) and 15 for other reasons (G-chemo, $n=9$; $R$ chemo, $\mathrm{n}=6$ ) (Online Supplementary Table S3). Post-progression survival in POD24 patients was similar in the two treatment arms at a median post-progression follow up of 22.6 months (Figure 2 and Online Supplementary Table S4).

\section{Survival and mortality by chemotherapy regimen, Follicular Lymphoma International Prognostic Index risk category, and treatment arm}

Fewer POD24 events occurred in G-chemo versus R-chemo patients across all three chemotherapy regimen groups; in each treatment arm, the highest rate of events was observed in those treated with CVP. POD24 events were more common in patients with a high FLIPI score when compared with those patients with an intermediate or low FLIPI score (Online Supplementary Tables S5 and S6).

\section{Histological transformation}

Histologically confirmed transformation to more aggressive lymphoma was observed in 35 patients: 13 $(2.2 \%)$ in the G-chemo arm and $22(3.7 \%)$ in the R-chemo arm, predominantly in the first 24 months $(n=30)$. In POD24 patients, transformation occurred in almost identical proportions of G-chemo- and R-chemo-treated patients [11 out of $57(19.3 \%)$ and 19 out of $98(19.4 \%)$ patients, respectively]. Of 30 patients $(19.4 \%)$ with transformation in the first 24 months, 16 died during study follow up ( $\mathrm{n}=8$ in each treatment arm). Of the 88 patients in the noPOD24 group with PD events after the 24-month time point, only $5(5.7 \%)$ had transformations [G-chemo, 2 of $40(5.0 \%)$; R-chemo, 3 of 48 (6.3\%)]. Cumulative incidence data and lymphoma types at transformation are presented in the Online Supplementary Results, Online Supplementary Table S7 and Online Supplementary Figure S2.

\section{New anti-lymphoma treatment}

New anti-lymphoma treatment (NALT) of any type was received by 100 of 155 patients $(64.5 \%)$ with a POD event in the first 24 months after randomization (G-chemo, $\mathrm{n}=37$; R-chemo, $\mathrm{n}=63)$ and by 24 of 88 patients $(27.3 \%)$ with a POD event later than the 24-month time point (G-chemo, $\mathrm{n}=11$; R-chemo, $\mathrm{n}=13$ ) (Online Supplementary Table S8). A cumulative incidence plot of time from PD to NALT for those with PD before and after 24 months showed that $49.7 \%$ (95\%CI: $41.3-57.5 \%$ ) of POD24 patients started NALT within three months of PD, compared with $19.4 \%$ (95\% CI: $11.2-29.3 \%)$ of those who progressed after 24 months (Online Supplementary Figure S3). Details of the type of NALT received after $\mathrm{PD}$ according to the timing of POD are provided in the Online Supplementary Results and Online Supplementary Table S8.

\section{Time-dependent mortality analysis}

Time-dependent regression analysis showed that, at any given time during study follow up, the risk of death was considerably greater following a POD24 event than in those without a POD24 event (stratified HR, 25.5; 95\% CI: 16.2-40.3\%) (Online Supplementary Table S9).

\section{Landmark analysis of overall survival and mortality}

In 110 patients with POD24 events who were still alive at the 24-month landmark (4 censored patients excluded), the OS rate at two years after the landmark was $82.4 \%$ (95\% CI: $74.2-91.3 \%$ ), compared with $98.2 \%$ (95\% CI: $97.1-99.2 \%)$ in 916 noPOD2 4 patients; the age-adjusted HR for OS in this 2-year window was 12.2 (95\%CI: 5.6-

Table 1. Baseline disease and patients' characteristics in POD24 and noPOD24 subgroups with at least 24 months' response follow up.

\begin{tabular}{lcc} 
Characteristic $^{\mathrm{a}}$ & $\begin{array}{c}\text { POD event within } \\
\text { first 24 months } \\
(\mathbf{n = 1 5 5 )}\end{array}$ & $\begin{array}{c}\text { No POD event } \\
\text { within first } \\
24 \text { months } \\
(\mathbf{n}=9116)\end{array}$ \\
Age, years & $58(31-84)$ & $59(23-85)$ \\
Aged $\geq 65$ years & $49(31.6)$ & $280(30.6)$ \\
\hline Male & $87(56.1)$ & $415(45.3)$ \\
Ann Arbor stage at diagnosis (n=1064) & & \\
I or II & $11 / 154(7.1)$ & $77 / 910(8.5)$ \\
III & $46 / 154(29.9)$ & $327 / 910(35.9)$ \\
IV & $97 / 154(63.0)$ & $506 / 910(55.6)$ \\
\hline Bone marrow involvement & & \\
(n=1063) & $80 / 155(51.6)$ & $468 / 908(51.5)$ \\
Grade of FL & & \\
1 & $57(36.8)$ & $353(38.5)$ \\
2 & $63(40.6)$ & $437(47.7)$ \\
$3^{\text {a }}$ & $35(22.6)$ & $117(12.8)$ \\
Other (incl. missing) & 0 & $9(1.0)$ \\
\hline
\end{tabular}

Time from diagnosis to randomization, months $(\mathrm{n}=1068) \quad 1.35(0.0-83.5) \quad 1.45(0.1-129.3)$

\begin{tabular}{lcc} 
& $(\mathrm{n}=154)$ & $(\mathrm{n}=914)$ \\
FLIPI & & \\
Low $(0-1)$ & $25(16.1)$ & $192(21.0)$ \\
Intermediate (2) & $46(29.7)$ & $354(38.6)$ \\
High $(\geq 3)$ & $84(54.2)$ & $370(40.4)$ \\
FLIPI-2 (n=1043) & & \\
Low (0) & $6 / 153(3.9)$ & $93 / 890(10.4)$ \\
Intermediate (1-2) & $68 / 153(44.4)$ & $452 / 890(50.8)$ \\
High $(\geq 3)$ & $79 / 153(51.6)$ & $345 / 890(38.8)$ \\
\hline Elevated serum lactate dehydrogenase & & \\
$>$ ULN & $79 / 154(51.3)$ & $248 / 912(27.2)$ \\
$>1.5 \times$ ULN & $26 / 154(16.9)$ & $51 / 912(5.6)$ \\
$>2 \times$ ULN & $10 / 154(6.5)$ & $18 / 912(2.0)$ \\
Investigator-assessed SPD & 6531 & 5128.5 \\
of $\leq 6$ target lesions, mm ${ }^{2}$ & $(514.0-80,720.0)$ & $(235.2-62,725.0)$ \\
\hline $\begin{array}{l}\text { Bulky disease } \geq 7 \mathrm{~cm} \\
(\mathrm{n}=1069)\end{array}$ & $79 / 155(51.0)$ & $392 / 914(42.9)$ \\
& &
\end{tabular}

\begin{tabular}{lcc}
$\begin{array}{l}\text { Randomized antibody treatment } \\
\text { Obinutuzumab } \\
\text { Rituximab }\end{array}$ & $57(36.8)$ & $478(52.2)$ \\
\hline $\begin{array}{llc}\text { Allocated chemotherapy treatment } \\
\text { Bendamustine }\end{array}$ & $98(63.2)$ & $438(47.8)$ \\
CHOP & $76(49.0)$ & $526(57.4)$ \\
CVP & $55(35.5)$ & $305(33.3)$ \\
\hline
\end{tabular}

n: number; CHOP: cyclophosphamide, doxorubicin, vincristine+prednisone; CVP: cyclophosphamide, vincristine+prednisone; FL: follicular lymphoma; FLIPI: Follicular Lymphoma International Prognostic Index; POD: progressive disease or death due to progressive disease; POD24: progressive disease or death due to progressive disease events in the 24 months after randomization; SPD: sum of products of 2 longest perpendicular dimensions for $\leq 6$ target lesions; ULN: upper limit of normal. ${ }^{a}$ Data are $n$ (\%), n/N (\%), or median (range). ${ }^{\mathrm{b}} 18$ patients were randomized to study treatment after being assessed as stage II or above by the investigators, meeting study eligibility criteria, but were reassessed as stage I after medical review. Staging data were missing for 7 patients (POD24, $n=1$; noPOD24, $n=6$ ) 
$26.5 \%$ ) (Figure 3D). KM survival plots after the 6-, 12-, and 18-month landmarks showed that the risk of death in patients whose disease progressed was higher the earlier progression occurred (Figure 3A-D). This is also shown by the corresponding mortality rates after progression during the selected time windows $(0-6,>6-12,>12-18$, and $>18$ 24 months from randomization) (Table 2); rates during each time window were similar for G-chemo- and Rchemo-treated patients (Online Supplementary Table S10).

\section{Discussion}

This analysis of updated data from the GALLIUM study confirmed the findings of prior studies ${ }^{4,10,11}$ showing that early disease progression in patients with FL is associated with a poor prognosis. The proportion of POD24 patients surviving at two years after the 24-month landmark was $82.4 \%$ (although $26 \%$ of POD24 patients had died before the 2-year landmark), whereas for the noPOD24 group, the proportion surviving at this time point was $98.2 \%$. Irrespective of treatment arm, patients who had PD in the 24 months after randomization were also much more likely to receive additional lymphoma therapy within three months of progression than patients who had PD later. By all of these measures, we confirm the adverse outlook associated with a POD24 event.

Treatment with G-chemo was associated with a marked reduction in the rate of POD24 events relative to $\mathrm{R}$-chemo and these data therefore support the superiority of the G-chemo regimen seen in the GALLIUM primary analysis. ${ }^{9}$ Notably, there was little difference in the proportion of patients in each treatment arm with PD after the 24-month time point, indicating that, to date, the protective effect of G-chemo against early progression has not been counterbalanced by any increased risk of later progression. With small numbers of deaths in both arms and comparatively short follow up (approximately 3.5 years), it should be noted that, despite a reduced risk of POD24 with G-chemo, we have not yet seen an impact on

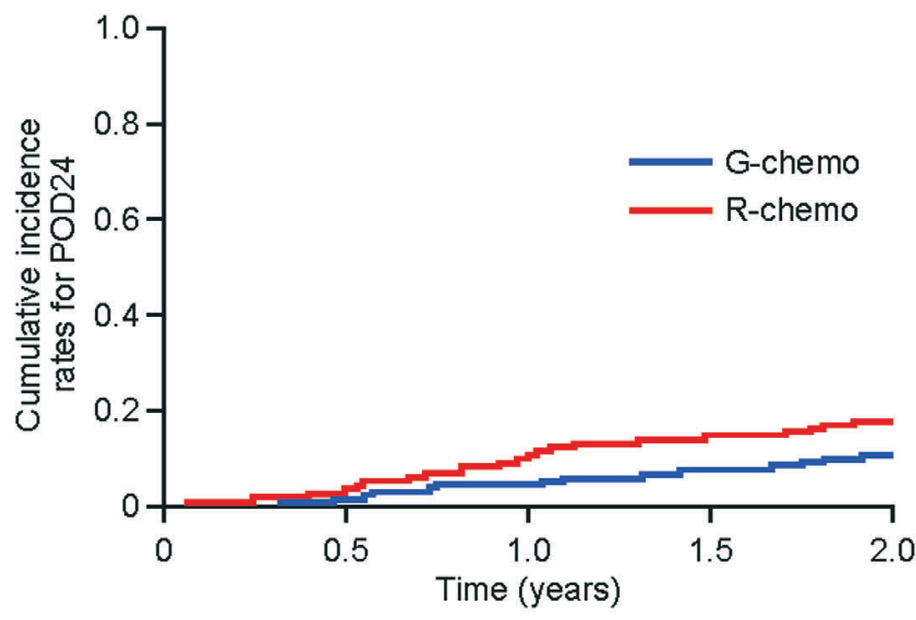

\begin{tabular}{|c|c|c|}
\hline & G-chemo $(n=601)$ & R-chemo $(n=601)$ \\
\hline \multicolumn{3}{|l|}{ In 24 months post-randomization: } \\
\hline PFS events, $n(\%)$ & $71(11.8)$ & $107(17.8)$ \\
\hline POD events, ${ }^{a}(\%)$ & $57(9.5)$ & $98(16.3)$ \\
\hline Deaths not due to $P D, n(\%)$ & $14(2.3)$ & $9(1.5)$ \\
\hline $\begin{array}{l}\text { 2-year cumulative incidence } \\
\text { of } \mathrm{POD} 24 \text { events }(95 \% \mathrm{Cl})\end{array}$ & $0.10(0.08-0.12)$ & $0.17(0.14-0.20)$ \\
\hline $\begin{array}{l}\text { Relative reduction in risk of POD } 24 \\
\text { events [(1-HR) } \times 100], \text { G-chemo vs } \\
\text { R-chemo, } \%(95 \% \mathrm{Cl})\end{array}$ & \multicolumn{2}{|c|}{$46.0(25.0-61.1)$} \\
\hline $\begin{array}{l}\text { Absolute risk of a PFS event in the } 24 \\
\text { months from randomization, } \%(95 \% \mathrm{Cl})\end{array}$ & $\begin{array}{c}12.5 \\
(10.1-15.6)\end{array}$ & $\begin{array}{c}18.9 \\
(15.9-22.4)\end{array}$ \\
\hline $\begin{array}{l}\text { Relative reduction in risk of PFS events in } \\
24 \text { months post-randomization } \\
\text { (G-chemo vs R-chemo), } \%(95 \% \mathrm{Cl})\end{array}$ & \multicolumn{2}{|c|}{$33.9(12.8-49.8)$} \\
\hline
\end{tabular}

Figure 1. Progression-free survival (PFS) and progressive disease or death due to progressive disease (POD) events in the 24 months after randomization (POD24) by treatment arm. The table below the graph shows the number of PFS and POD events occurring in the 24 months post-randomization, along with the risk of these events. $n$ : number; PD: progressive disease; $\mathrm{Cl}$ : confidence interval; HR: hazard ratio; G-chemo: obinutuzumab plus chemotherapy; R-chemo: rituximab plus chemotherapy. ${ }^{\text {aAll }} 155$ patients had PD. ${ }^{\mathrm{b} A t} 24$ months after randomization, deaths from any cause had occurred in 26 (Gchemo) and 38 (R-chemo) patients.

Table 2. Post-progression mortality rates, stratified by time of progression, in the POD24 group ( $n=155)$.

\begin{tabular}{lcccccc}
\hline ifime of progression, months & $n$ & Deaths & \multicolumn{2}{c}{$\begin{array}{c}\text { Reason for death } \\
\text { PD }\end{array}$} & $\begin{array}{c}\text { Patient-years at risk } \\
\text { Other }\end{array}$ & $\begin{array}{c}\text { Deaths per 100 patient-years } \\
\text { (95\%Cl) }\end{array}$ \\
$0-6$ & 22 & 18 & 16 & 2 & 22.2 & $81.0(51.2-100)$ \\
$>6-12$ & 58 & 27 & 19 & 8 & 111.6 & $24.2(16.6-35.3)$ \\
$>12-18$ & 46 & 9 & 5 & 4 & 102.2 & $8.8(4.6-16.9)$ \\
$>18-24$ & 29 & 2 & 0 & 2 & 52.5 & $3.8(1.0-15.2)$
\end{tabular}

n: number; CI: confidence interval; PD: progressive disease; POD24: progressive disease or death due to progressive disease events in the 24 months after randomization. 
OS in the GALLIUM study. However, this is not unexpected with current follow up given the available salvage therapies.

Our results suggest that POD24 events are more common in patients treated with CVP compared with patients treated with $\mathrm{CHOP}$ or bendamustine. However, it is difficult to draw firm conclusions from these results, as patient numbers within the subgroups are low. Furthermore, statistical comparisons across chemotherapy regimens were not performed, as chemotherapy allocation was not randomized; rather the chemotherapy treatment was selected by the investigator, with all patients at each individual center receiving the same regimen.

As previously reported, fewer patients with FL in the Gchemo arm than in the R-chemo arm started NALT during GALLIUM (estimated proportions without NALT at three years: $87 \%$ and $81 \%$, respectively). ${ }^{9}$ However, the current analysis shows that patients who progressed early (within 24 months of randomization) were much more likely to receive NALT within three months of progression than patients who had PD later, irrespective of treatment arm.

Transformation to a more aggressive lymphoma at the time of PD occurred in just under $20 \%$ of those patients with POD24 events, with no discernible difference in these proportions by treatment arm. However, transformations appear to be much less frequent in patients with later progression; at the current data cutoff, only $5.7 \%$ of patients in the noPOD24 group experienced transformation.

The increase in mortality risk associated with POD24 in the current analysis (HR from stratified Cox regression analysis, 25.5) was much larger than in a recent pooled analysis of POD24 in 5453 patients from 13 clinical trials (HR for OS, 5.24) $)^{5}$ and the National LymphoCare Study (NLCS) analysis by Casulo et al. ${ }^{4}$ which reported an HR of 8.1 in an exploratory analysis that used the same method as in the current analysis, i.e. POD24 as a time-varying covariate and adjusting for FLIPI. Importantly, the follow-up duration in our analysis was shorter than in the NLCS (medians, 3.4 and 7 years, respectively); as the follow-up duration in GALLIUM increases, the HR value is likely to fall, as we showed that the risk of death is higher for patients with earlier progression. However, survival data for the first two years of follow up in our study look remarkably similar to the NLCS analysis. In GALLIUM, the proportion of POD24 patients surviving at two years after progression was $66 \%$ (95\% CI: 58.3-73.9\%) whereas for the noPOD24 group, the proportion surviving at two years after the 24-month landmark was $98.2 \%(95 \% \mathrm{CI}$ : 97.1-99.2); in NLCS, the corresponding proportions were 68\% (95\%CI: $58.2-76.3$ ) and 97\% (95\%CI: 94.6-98.1), respectively. ${ }^{4}$

Our findings also suggest that the earlier progression occurs, the greater the subsequent mortality risk. This subset of patients with FL in whom the disease follows a relatively aggressive clinical course may benefit from alternative initial therapies. Therefore, identifying these patients is crucial. The baseline factor in the current analysis most strongly associated with risk of POD24 was serum LDH level, in line with previous studies that have identified LDH as a prognostic factor for progression in patients with FL. ${ }^{12,13}$ Other factors associated with greater POD risk were FLIPI risk group, disease stage and histological grade, male sex, and treatment with CVP chemotherapy; although the extent of difference in $\mathrm{POD}$ risk accounted for by these was not as great as the influence of anti-CD20 antibody treatment.

Promising prospective identification of patients with a high risk of early PD events has been achieved by using the $\mathrm{m} 7$-FLIPI, a clinicogenetic risk model incorporating the impact of mutations in the genes EZH2, ARID1A, MEF2B, EP300, FOXO1, CREBBP, and CARD11. ${ }^{14}$ The $\mathrm{m} 7$-FLIPI had higher predictive accuracy for POD24 than either the FLIPI or another specific clinicogenetic risk model, the POD24 Prognostic Index (POD24-PI), in an analysis of 258 patients from two independent cohorts. ${ }^{10} \mathrm{~A}$ new 23 -gene expression model was also reported to predict early progression..$^{15}$ Additionally, TP53 mutation status has been evaluated as a prognostic factor in patients with FL and found to be significantly correlated with shorter PFS, OS,

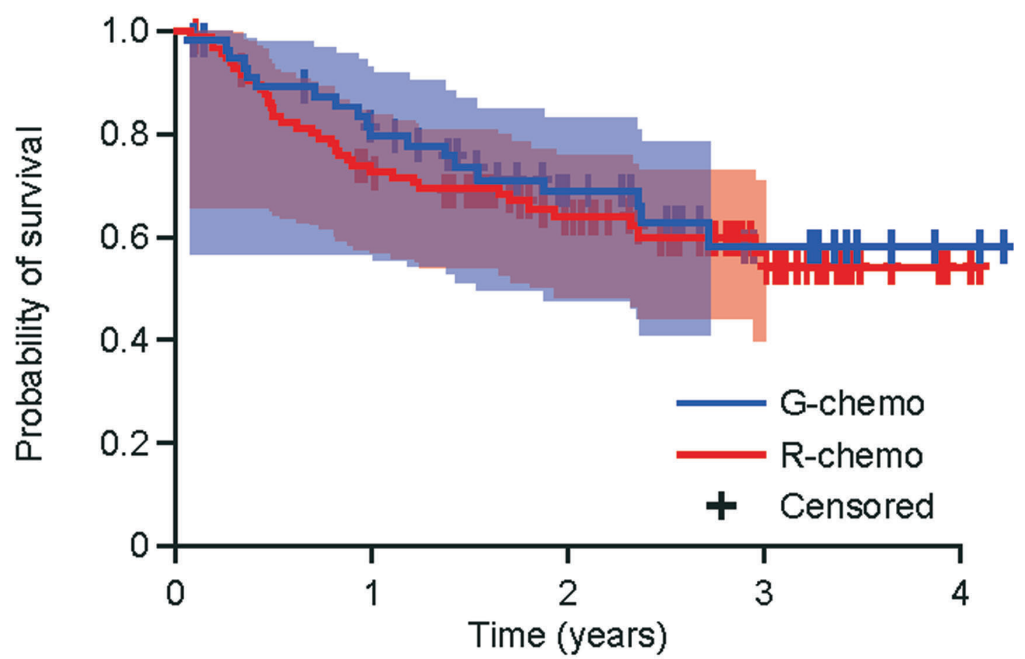

N. of patients at risk

$\begin{array}{rr}57 & 43 \\ - & 98\end{array}$

69
25

40
9

19
Figure 2. Overall survival in POD24 patients post-progression by treatment arm. Shaded sections of lines show $95 \%$ Hall-Wellner confidence bands for the period during which patients died. G-chemo: obinutuzumab plus chemotherapy; R-chemo: rituximab plus chemotherapy; N: number. POD24: progressive disease or death due to progressive disease events in the 24 months after randomization. 
and disease transformation. ${ }^{16,17}$ Interrogation of the GALLIUM dataset using m7-FLIPI and other potentially predictive indices is ongoing.

In conclusion, our results are consistent with previous studies that found a particularly poor outlook for patients with FL who suffer PD within 24 months of starting immunochemotherapy, and also further refine this obser- vation by showing that there is a gradation of prognosis even within the POD24 subset, with earlier events predicting more adverse outcomes. We observed that patients receiving G-based treatment have a much lower POD24 rate than those receiving R-based treatment; this supports the contention that more effective first-line treatment that reduces the POD24 rate may yield long-term benefit. Our
A

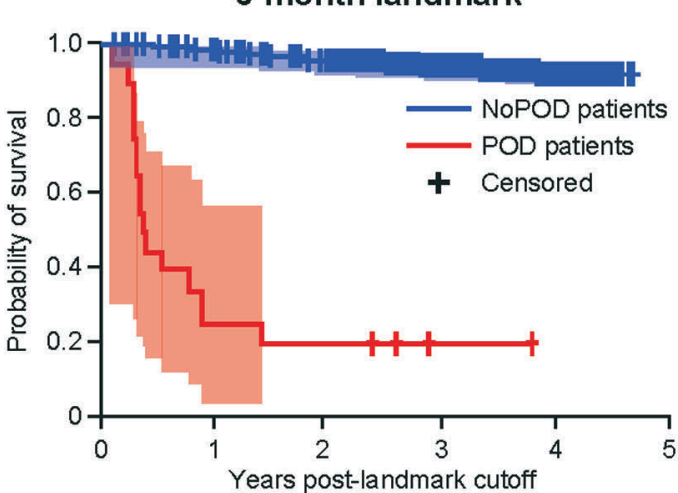

N. of patients at risk
B

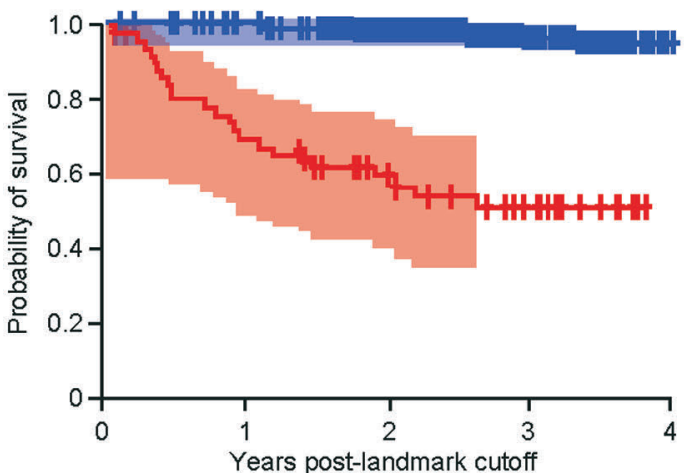

N. of patients at risk

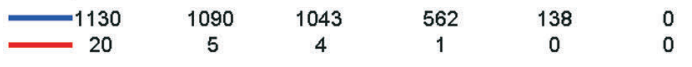

C

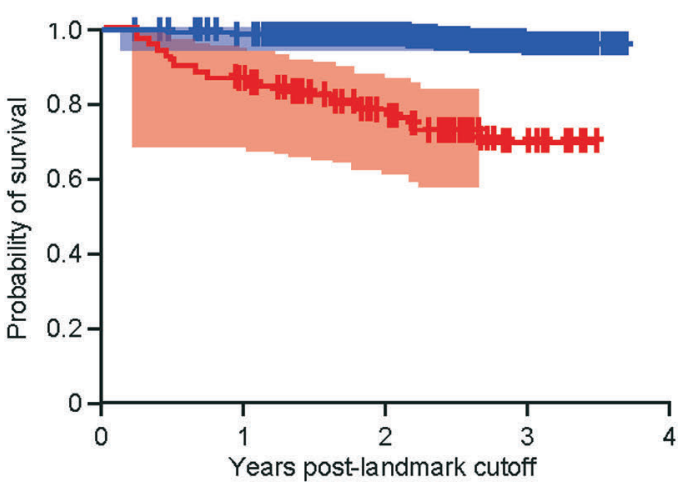

N. of patients at risk

$\begin{array}{ccccc}-1040 & 1014 & 816 & 335 & 7 \\ -61 & 40 & 24 & 12 & 0\end{array}$

D

24-month landmark

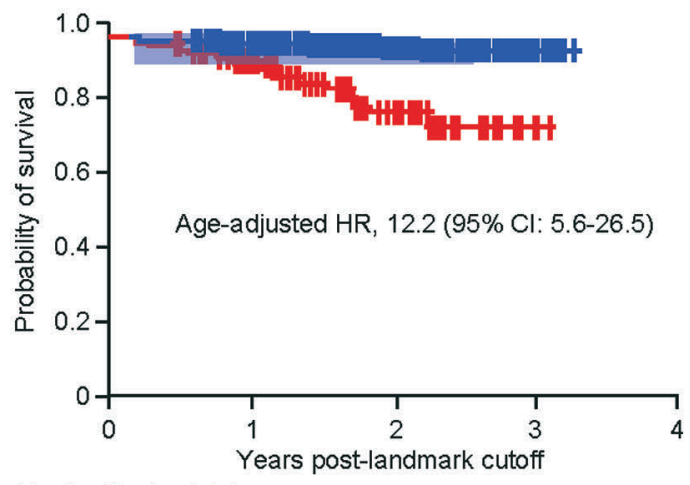

$\mathrm{N}$. of patients at risk

\begin{tabular}{cccccccccc}
-969 & 950 & 516 & 562 & 0 & -916 & 751 & 306 & 7 & 0 \\
\hline 90 & 73 & 41 & 11 & 0 & -110 & 80 & 37 & 0 & 0
\end{tabular}

\begin{tabular}{lcccc}
\hline & \multicolumn{2}{c}{ POD } & \multicolumn{3}{c}{ noPOD } \\
\hline Landmark & $\begin{array}{c}\text { OS (\%) at } \\
2 \text { years } \\
\text { post-landmark }\end{array}$ & $95 \% \mathrm{Cl}$ & $\begin{array}{c}\text { OS (\%) at } \\
2 \text { years } \\
\text { post-landmark }\end{array}$ & $95 \% \mathrm{Cl}$ \\
\hline 6 months & 20 & $2.5-37.5$ & 95.8 & $94.6-97.0$ \\
12 months & 58.4 & $45.5-71.3$ & 97.6 & $96.7-98.6$ \\
18 months & 76.5 & $67.0-86.0$ & 97.8 & $97.0-98.9$ \\
24 months & 82.4 & $74.2-91.34$ & 98.2 & $97.1-99.2$ \\
\hline
\end{tabular}

Figure 3. Landmark overall survival (OS) analysis, comparing patients with progressive disease or death due to progressive disease (POD) before the landmark and patients with noPOD. (A) 6-month, (B) 12-month, (C) 18-month, and (D) 24-month landmarks. The shaded sections of lines show 95\% Hall-Wellner confidence bands for the period during which patients died. The table below the graph shows 2 -year OS estimates (with $95 \% \mathrm{Cl}$ ) at each landmark. N: number; Cl: confidence interval; HR: hazard ratio. 
analysis also adds to the weight of data supporting the use of POD24 rate as an efficacy end point for future clinical trials in patients with previously untreated FL.

\section{Acknowledgments}

The authors would like to thank all of the patients who participated in the GALLIUM study, and acknowledge all of the study investigators and their staff as well as the GALLIUM study team. Third-party medical writing assistance, under the direction of John Seymour, was provided by Roger Nutter, Scott Malkin and Helen Cathro of Gardiner-Caldwell Communications, and was funded by F. Hoffmann-La Roche Ltd.

\section{References}

1. Marcus R, Imrie $\mathrm{K}$, Belch $\mathrm{A}$, et al. CVP chemotherapy plus rituximab compared with CVP as first-line treatment for advanced follicular lymphoma. Blood. 2005;105(4):1417-1423

2. Press OW, Unger JM, Rimsza LM, et al. Phase III randomized intergroup trial of CHOP plus rituximab compared with CHOP chemotherapy plus (131)iodine-tositumomab for previously untreated follicular non-Hodgkin lymphoma: SWOG S0016. J Clin Oncol. 2013;31(3):314-320.

3. Rummel MJ, Niederle N, Maschmeyer G, et al. Bendamustine plus rituximab versus $\mathrm{CHOP}$ plus rituximab as first-line treatment for patients with indolent and mantle-cell lymphomas: an open-label, multicentre, randomised, phase 3 non-inferiority trial. Lancet. 2013;381(9873):1203-1210.

4. Casulo C, Byrtek M, Dawson KL, et al. Early relapse of follicular lymphoma after rituximab plus cyclophosphamide, doxorubicin, vincristine, and prednisone defines patients at high risk for death: an analysis from the National LymphoCare Study. J Clin Oncol. 2015;33(23):2516-2522.

5. Casulo C, Le-Rademacher J, Dixon J, et al. Validation of POD24 as a robust early clinical endpoint of poor survival in follicular lymphoma: results from the Follicular Lymphoma Analysis of Surrogacy Hypothesis (FLASH) investigation using individual data from 5,453 patients on 13 clinical trials. Blood. 2017;130(Suppl 1):412

6. Herter $S$, Herting F, Mindigl $O$, et al Preclinical activity of the type II CD20 antibody GA101 (obinutuzumab) compared with rituximab and ofatumumab in vitro and in xenograft models. Mol Cancer Ther 2013;12(10):2031-2042.

7. Mössner E, Brünker $\mathrm{P}$, Moser $\mathrm{S}$, et al Increasing the efficacy of CD20 antibody therapy through the engineering of a new type II anti-CD20 antibody with enhanced direct and immune effector cell-mediated Bcell cytotoxicity. Blood. 2010; 115(22):43934402

8. Hiddemann W, Barbui AM, Canales MA, et al. Immunochemotherapy with obinutuzumab or rituximab for previously untreated follicular lymphoma in the GAL LUM study: influence of chemotherapy on efficacy and safety. J Clin Oncol. 2018; 36(23):2395-2404.

9. Marcus R, Davies A, Ando K, et al. Obinutuzumab for the first-line treatment of follicular lymphoma. N Engl J Med. 2017:377(14):1331-1344

10. Jurinovic V, Kridel R, Staiger AM, et al. Clinicogenetic risk models predict early progression of follicular lymphoma after firstline immunochemotherapy. Blood. 2016;128(8):1112-1120.

11. Maurer MJ, Bachy E, Ghesquières $\mathrm{H}$, et al. Early event status informs subsequent outcome in newly diagnosed follicular lymphoma. Am J Hematol. 2016;91(11):10961101.
12. Montoto S, Lopez-Guillermo A, Ferrer A, et al. Survival after progression in patients with follicular lymphoma: analysis of prognostic factors. Ann Oncol. 2002:13(4):525-530.

13. Murakami S, Kato H, Yamamoto K, et al. Combination of high serum lactate dehydrogenase levels at the time of first diagnosis and progression predicts early lymphoma related death in patients with follicular lymphoma receiving R-CHOP regimen. Blood. 2014;124(21):2972.

14. Pastore A, Jurinovic V, Kridel R, et al. Integration of gene mutations in risk prognostication for patients receiving first-line immunochemotherapy for follicular lymphoma: a retrospective analysis of a prospective clinical trial and validation in a population-based registry. Lancet Oncol. 2015;16(9):1111-1122.

15. Huet S, Tesson B, Jais JP, et al. A geneexpression profiling score for prediction of outcome in patients with follicular lymphoma: a retrospective training and validation analysis in three international cohorts. Lancet Oncol. 2018;19(4):549-561.

16. O'Shea D, O'Riain C, Taylor C, et al. The presence of TP53 mutation at diagnosis of follicular lymphoma identifies a high-risk group of patients with shortened time to disease progression and poorer overall survival. Blood. 2008;112(8):3126-3129.

17. Kridel R, Chan FC, Mottok A, et al Histological transformation and progression in follicular lymphoma: a clonal evolution study. PLoS Med. 2016;13(12):e1002197. 